\title{
SEMIGROUP-LIKE PROPERTY OF MITTAG-LEFFLER FUNCTION
}

\section{Bambang Hendriya Guswanto}

\author{
Analysis and Algebra Laboratory, Department of Mathematics \\ Faculty of Mathematics and Natural Sciences \\ Jenderal Soedirman University \\ Email : bambanghg_unsoed@yahoo.com
}

\begin{abstract}
In this article, we discuss the semigroup-like property of MittagLeffler function which is used to solve the initial value problem of an ordinary differential equation involving Caputo fractional derivative.
\end{abstract}

Keywords : Semigroup-like property, Mittag-Leffler function, Caputo fractional derivative.

ABSTRAK. Pada artikel ini, kita membahas sifat mirip semigroup dari fungsi Mittag-Leffler yang digunakan untuk menyelesaikan masalah nilai awal suatu persamaan diferensial biasa yang menggunakan turunan fraksional Caputo.

Kata kunci : sifat mirip semigroup, fungsi Mittag-Leffler, turunan fraksional Caputo.

\section{INTRODUCTION}

Let us consider the following initial value problem

$$
\begin{aligned}
D_{t} v(t) & =\lambda v(t)+f(t), \quad t>0, \\
v(0) & =v_{0}
\end{aligned}
$$

where $\lambda \in \mathbb{C}$ and $f$ is a complex function defined in $(0, \infty)$. We can find that if $v:[0, \infty) \rightarrow \mathbb{C}$ is a continuous function solving the problem (1) then it is given uniquely by

$$
v(t)=v_{0} e^{\lambda t}+\int_{0}^{t} e^{\lambda(t-s)} f(s) d s, t>0 .
$$


If $f=0$, we have the homogeneous case of the problem (1) and $v(t)=v_{0} e^{\lambda t}$ is the solution to the case. Moreover, it is not difficult to show that, for $s \geq 0, e^{\lambda(t+s)}$ is the solution to the problem (1) in case $v_{0}=e^{\lambda s}$ satisfying

$$
e^{\lambda(t+s)}=e^{\lambda t} e^{\lambda s}
$$

The identity (2) is called semigroup property of exponential function.

We now consider the initial value problem

$$
\begin{aligned}
D_{t}^{\alpha} v(t) & =\lambda v(t)+f(t), \quad t>0, \\
v(0) & =v_{0}
\end{aligned}
$$

where $\lambda \in \mathbb{C}, f$ is a complex function defined in $(0, \infty)$, and $D_{t}^{\alpha}$ is the Caputo fractional derivative of order $\alpha$ defined by

$$
D_{t}^{\alpha} v(t)=\int_{0}^{t} \frac{(t-s)^{-\alpha}}{\Gamma(1-\alpha)} D_{s} v(s) d s, 0<\alpha<1,
$$

where $\Gamma$ is Gamma function. Here, we review specially the application of MittagLeffler function to solve the problem (3) and derive semigroup-like property of the function. More generally, one can refer to (Guswanto, 2015) for similar discussion in abstract problem employing functional analysis theory.

This article is composed of three sections. In the second section, we introduce briefly Mittag-Leffler function and its properties. In the last section, we show the semigrouplike property of Mittag-Leffler function.

\section{MITTAG-LEFFLER FUNCTION}

In this section, we introduce Mittag-Leffler function defined by

$$
E_{\alpha, \beta}(z)=\sum_{n=0}^{\infty} \frac{z^{n}}{\Gamma(\alpha n+\beta)}, \alpha, \beta>0, z \in \mathbb{C} .
$$

This function is entire and can be represented in the integral form 


$$
E_{\alpha, \beta}(z)=\frac{1}{2 \pi i} \int_{\Gamma_{r, \omega}} \frac{e^{\zeta} \zeta^{\alpha-\beta}}{\zeta^{\alpha}-z} d \zeta, \quad \zeta^{1 / \alpha} \in \Gamma_{r, \omega}^{-}
$$

and

$$
E_{\alpha, \beta}(z)=\frac{1}{\alpha} z^{(1-\beta) / \alpha} e^{1 / \alpha}+\frac{1}{2 \pi i} \int_{\Gamma_{r, \omega}} \frac{e^{\zeta} \zeta^{\alpha-\beta}}{\zeta^{\alpha}-z} d \zeta, \quad \zeta^{1 / \alpha} \in \Gamma_{r, \omega}^{+}
$$

where

$$
\Gamma_{r, \omega}=\{z \in \mathbb{C}:|\arg (z)|=\omega,|z| \geq r\} \cup\{z \in \mathbb{C}:|\arg (z)| \leq \omega,|z|=r\}
$$

and $\Gamma_{r, \omega}^{-}\left(\Gamma_{r, \omega}^{+}\right)$is the area lying on the left (right) hand side of the contour $\Gamma_{r, \omega}$ with $0<\alpha<2, \beta \in \mathbb{C}, r>0$, and $\pi / 2<\omega<\pi$.

We now give estimates for Mittag-Leffler function. For $\mu \in \mathbb{R}$ such that $\pi \alpha / 2<\mu<$ $\min \{\pi, \pi \alpha\}$, there are $D_{i} \in \mathbb{R}, i=1,2,3$ such that, for $|z| \geq 0,|\arg (z)| \leq \mu$,

$$
\left|E_{\alpha, \beta}(z)\right| \leq D_{1}(1+|z|)^{(1-\beta) / \alpha} e^{\operatorname{Re}\left(z^{1 / \alpha}\right)}+\frac{D_{2}}{1+|z|}
$$

and, for $|z| \geq 0, \mu \leq|\arg (z)| \leq \pi$,

$$
\left|E_{\alpha, \beta}(z)\right| \leq \frac{D_{3}}{1+|z|}
$$

We also have the Laplace transform of Mittag-Leffler function, that is

$$
\mathcal{L}\left(t^{\beta-1} E_{\alpha, \beta}\left(\lambda t^{\alpha}\right)\right)(\xi)=\frac{\xi^{\alpha-\beta}}{\xi^{\alpha}-\lambda}, \operatorname{Re}(\xi)>|\lambda|^{1 / \alpha},
$$

where Laplace transform is defined by

$$
\mathcal{L}(f(t))(\xi)=\int_{0}^{\infty} e^{-\xi t} f(t) d t, f \in L^{1}((0, \infty)) .
$$

Furthermore, we can derive some identities involving Mittag-Leffler function, those are 


$$
D_{t} E_{\alpha}\left(\lambda t^{\alpha}\right)=\lambda t^{\alpha-1} E_{\alpha, \alpha}\left(\lambda t^{\alpha}\right), \quad t>0
$$

and

$$
D_{t}^{\alpha} E_{\alpha}\left(\lambda t^{\alpha}\right)=\lambda E_{\alpha}\left(\lambda t^{\alpha}\right), \quad t>0
$$

We refer to (Kilbas et al., 2006) or (Podlubny, 1999) for more details concerning the fractional derivative and Mittag-Leffler function.

\section{RESULTS AND DISCUSSION}

We consider again the problem (3). We can find that the Laplace transform of (3) is

$$
\xi^{\alpha} \mathcal{L}(v)(\xi)-\xi^{\alpha-1} v_{0}=\lambda \mathcal{L}(v)(\xi)+\mathcal{L}(f)(\xi) .
$$

Then

$$
\mathcal{L}(v)(\xi)=\frac{\xi^{\alpha-1}}{\xi^{\alpha}-\lambda} v_{0}+\frac{\xi^{\alpha-\alpha}}{\xi^{\alpha}-\lambda} \mathcal{L}(f)(\xi), \quad \operatorname{Re}(\xi)>|\lambda|^{1 / \alpha} .
$$

By the invers of Laplace transform, we have

$$
v(t)=E_{\alpha}\left(\lambda t^{\alpha}\right) v_{0}+\int_{0}^{t}(t-s)^{\alpha-1} E_{\alpha, \alpha}\left(\lambda(t-s)^{\alpha}\right) f(s) d s, t>0 .
$$

By the uniqueness of the invers of Laplace transform, the solution $v(t)$ is unique.

We next prove that Mittag-Leffler function does not satisfy semigroup property. In order to do this, we prove that $E_{\alpha}\left(\lambda(t+s)^{\alpha}\right)$ solves the problem

$$
\begin{aligned}
D_{t}^{\alpha} v(t) & =\lambda v(t)-F(t), t>0, \\
v(0) & =E_{\alpha}\left(\lambda s^{\alpha}\right)
\end{aligned}
$$

where

$$
F(t)=\int_{0}^{s} \frac{(t+s-r)}{\Gamma(1-\alpha)} D_{r} E_{\alpha}\left(\lambda r^{\alpha}\right) d r, t>0 .
$$

We show first that the complex function $F$ is defined in $(0, \infty)$ for any $\lambda \in \mathbb{C}$ and $s>0$. We suppose that $\pi \alpha / 2<\phi<\min \{\pi, \pi \alpha\}$. Observe that, by (5) and (7), for $\lambda \in \mathbb{C}$ with $\phi \leq|\arg (\lambda)| \leq \pi$, 


$$
\begin{aligned}
F(t) & \leq \frac{|\lambda|}{\Gamma(1-\alpha)} \int_{0}^{s}(t+s-r)^{-\alpha} r^{\alpha-1}\left|E_{\alpha, \alpha}\left(\lambda r^{\alpha}\right)\right| d r \\
& \leq \frac{|\lambda| D_{3}}{\Gamma(1-\alpha)} \int_{0}^{s}(t+s-r)^{-\alpha} r^{\alpha-1}\left(1+|\lambda| r^{\alpha}\right)^{-1} d r \\
& \leq \frac{|\lambda| D_{3}}{\Gamma(1-\alpha)} \int_{0}^{s}(s-r)^{-\alpha} r^{\alpha-1} d r \\
& =\frac{|\lambda| D_{3} B(\alpha, 1-\alpha)}{\Gamma(1-\alpha)}, t>0
\end{aligned}
$$

and, by (4) and (7), for $\lambda \in \mathbb{C}$ with $|\arg (\lambda)| \leq \phi$,

$$
\begin{aligned}
F(t) \leq & \frac{|\lambda| D_{2} B(\alpha, 1-\alpha)}{\Gamma(1-\alpha)} \\
& +\frac{|\lambda| D_{1}}{\Gamma(1-\alpha)} \int_{0}^{s}(t+s-r)^{-\alpha} r^{\alpha-1}\left(1+|\lambda| r^{\alpha}\right)^{\frac{1-\alpha}{\alpha}} e^{r \operatorname{Re}\left(\lambda^{1 / \alpha}\right)} d r \\
\leq & \frac{|\lambda| D_{2} B(\alpha, 1-\alpha)}{\Gamma(1-\alpha)} \\
& +\frac{|\lambda|\left(1+|\lambda| s^{\alpha}\right)^{\frac{1-\alpha}{\alpha}} D_{1}}{\Gamma(1-\alpha)} \int_{0}^{s}(s-r)^{-\alpha} r^{\alpha-1} e^{r \operatorname{Re}\left(\lambda^{1 / \alpha}\right)} d r \\
= & \frac{|\lambda| D_{2} B(\alpha, 1-\alpha)}{\Gamma(1-\alpha)}+\frac{|\lambda|\left(1+|\lambda| s^{\alpha}\right)^{\frac{1-\alpha}{\alpha}} D_{1} D_{4}}{\Gamma(1-\alpha)}, t>0,
\end{aligned}
$$

where

$$
B(a, b)=\int_{0}^{1} r^{a-1}(1-r)^{b-1} d r, \quad a, b>0,
$$

is Beta function and

$$
D_{4}=\int_{0}^{1}(1-\rho)^{-\alpha} \rho^{\alpha-1} e^{s \rho \operatorname{Re}\left(\lambda^{1 / \alpha}\right)} d \rho
$$


Thus the function $F$ is defined in $(0, \infty)$ for any $\lambda \in \mathbb{C}$ and $s>0$.

Next, consider that

$$
\begin{aligned}
D_{t}^{\alpha} E_{\alpha}\left(\lambda(t+s)^{\alpha}\right) & =\int_{0}^{t} \frac{(t-\tau)^{-\alpha}}{\Gamma(1-\alpha)} D_{\tau} E_{\alpha}\left(\lambda(\tau+s)^{\alpha}\right) d \tau \\
& =\int_{s}^{t+s} \frac{(t+s-r)^{-\alpha}}{\Gamma(1-\alpha)} D_{r} E_{\alpha}\left(\lambda r^{\alpha}\right) d r \\
& =\int_{0}^{t+s} \frac{(t+s-r)^{-\alpha}}{\Gamma(1-\alpha)} D_{r} E_{\alpha}\left(\lambda r^{\alpha}\right) d r \\
& -\int_{0}^{s} \frac{(t+s-r)^{-\alpha}}{\Gamma(1-\alpha)} D_{r} E_{\alpha}\left(\lambda r^{\alpha}\right) d r \\
& =D_{t+s}^{\alpha} E_{\alpha}\left(\lambda(t+s)^{\alpha}\right)-F(t)
\end{aligned}
$$

By (8), the equation (11) is reduced to

$$
D_{t}^{\alpha} E_{\alpha}\left(\lambda(t+s)^{\alpha}\right)=\lambda E_{\alpha}\left(\lambda(t+s)^{\alpha}\right)-F(t) .
$$

It follows that $v(t)=E_{\alpha}\left(\lambda(t+s)^{\alpha}\right)$ solves the problem (10) and, by (9), it is uniquely represented by

$$
E_{\alpha}\left(\lambda(t+s)^{\alpha}\right)=E_{\alpha}\left(\lambda t^{\alpha}\right) E_{\alpha}\left(\lambda s^{\alpha}\right)-\int_{0}^{t} \tau^{\alpha-1} E_{\alpha, \alpha}\left(\lambda \tau^{\alpha}\right) F(t-\tau) d \tau
$$

and, moreover, by (7), it becomes

$$
\begin{aligned}
E_{\alpha}\left(\lambda(t+s)^{\alpha}\right)= & E_{\alpha}\left(\lambda t^{\alpha}\right) E_{\alpha}\left(\lambda s^{\alpha}\right) \\
& -\lambda \int_{0}^{t} \int_{0}^{s} \frac{(t+s-\tau-r)^{-\alpha}}{\Gamma(1-\alpha)}(\tau r)^{\alpha-1} E_{\alpha, \alpha}\left(\lambda \tau^{\alpha}\right) E_{\alpha, \alpha}\left(\lambda r^{\alpha}\right) d r d \tau .
\end{aligned}
$$

We call the identity (12) semigroup-like property of Mittag-Leffler function. 


\section{REFERENCES}

Guswanto, B. H., On the Properties of Solution Operators of Fractional Evolution

Equations, Journal of Fractional Calculus and Applications, Vol. 6(1) (2015), 131159.

Kilbas, A. A., Srivastava, H. M., and Trujilo, J. J., Theory and Application of Fractional Differential Equations, North Holland Mathematics Studies, Elsevier, 2006.

Podlubny, I, Fractional Differential Equations, Academic Press 198, 1999. 